\title{
Multiple Time Analyticity of a Quantum Statistical State Satisfying the KMS Boundary Condition
}

By

\author{
Huzihiro ARAKI
}

\begin{abstract}
A multiple time expectation $\varphi\left(A B_{1}\left(t_{1}\right) \cdots B_{n}\left(t_{n}\right)\right)$ in a stationary state $\varphi$ satisfying the KMS boundary condition is studied. It is found to be holomorphic in a simplicial tube domain $0<\operatorname{Im} t_{1}<\operatorname{Im} t_{2}<\cdots<\operatorname{Im} t_{n}<\beta$, continuous and bounded in the closure and the expectation of cyclic permutation of operators are obtained as its values on various distinguished boundaries of the domain.
\end{abstract}

\section{$\S 1$. Introduction}

The Gibbs ensemble in quantum statistical mechanics satisfies the Kubo-Martin-Schwinger (KMS) boundary condition and a general property of such a state has been discussed by several authors [1], [2], [3], [4]. In this paper we shall study the analyticity of $\varphi\left(A B_{1}\left(t_{1}\right) \cdots B_{n}\left(t_{n}\right)\right)$ in $t_{1} \cdots t_{n}$. The main theorem is Theorem 3.1 and 3.3 of section 3 .

In passing, it is shown by the analyticity method that the center of the representing algebra is time translation invariant. It is also pointed out that the KMS boundary condition holds for the weak closure, which will be used in [4].

\section{§. The KMS Boundary Condition and Analyticity}

We shall discuss an analyticity tube domain for single time expectation function in this section. We also give a proof that the center of the representative algebra is elementwise time translation invariant. 
Let $\mathfrak{A}$ be a $C^{*}$ algebra, $\tau(t)$ be a one parameter group of automorphisms of $\mathfrak{A}$, continuous in $t$, and $\phi$ be a state of $\mathfrak{A}$ invariant under $\tau(t)$ :

$$
\varphi(A)=\varphi(\tau(t) A), \quad A \in \mathfrak{U} \text {. }
$$

Definition 2.1. $\varphi$ satisfies the KMS boundary condition if

$$
\begin{gathered}
\int \varphi(A \tau(t) B) \tilde{f}_{0}(t) \mathrm{d} t=\int \phi([\tau(t) B] A) \tilde{f}_{\beta}(t) \mathrm{d} t, \\
\tilde{f}_{\alpha}(t)=\int_{-\infty}^{\infty} f(p) e^{-i p t t_{\top} \phi} \mathrm{d} p
\end{gathered}
$$

for arbitrary two elements $A$ and $B$ of $\mathfrak{A}$ and for arbitrary function $f$ in the class $\mathscr{D}$.

Lemma 2. 2. If $\phi$ is $\tau(t)$ invariant and satisfies the KMS boundary condition, and $A$ and $B$ are elements of $\mathfrak{A}$, then there exists a function $\mathrm{F}(\zeta)$ of a complex variable $\zeta$ such that

(1) $\mathrm{F}$ is continuous and bounded for $0 \leq \operatorname{Im} \zeta \leq \beta$.

(2) $\mathrm{F}$ is holomorphic for $0<\operatorname{Im} \zeta<\beta$.

(3) For real $t$,

$$
\mathrm{F}(t)=\varphi(A \tau(t) B), \quad \mathrm{F}(t+i \beta)=\varphi([\tau(t) B] A) .
$$

Proof. We note that a representation $\pi_{\varphi}$ of $\mathfrak{A}$ on a Hilbert space $H_{\varphi}$, a cyclic vector $\Omega_{\varphi}$ and a continuous one parameter group of unitary operator $U_{\varphi}(t)$ are uniquely determined by the relation

$$
\begin{gathered}
\varphi(A)=\left(\Omega_{\varphi}, \pi_{\varphi}(A) \Omega_{\varphi}\right) \\
U_{\varphi}(t) \pi_{\varphi}(A) \Omega_{\varphi}=\pi_{\varphi}(\tau(t) A) \Omega_{\varphi} .
\end{gathered}
$$

In particular,

$$
\varphi(A \tau(t) B)=\left(\Omega_{\varphi}, \pi_{\varphi}(A) U_{\varphi}(t) \pi_{\varphi}(B) \Omega_{\varphi}\right)
$$

is a Fourier transform of a finite complex measure $\mu_{0}$ :

$$
\varphi(A \tau(t) B)=\int e^{i p t} \mathrm{~d} \mu_{0}(p) .
$$

Similarly

$$
\mathcal{P}([\tau(t) B] A)=\int e^{i \not t} \mathrm{~d} \mu_{\beta}(p)
$$


A complex finite measure can be considered as a dual to the Banach space $\mathcal{C}_{0}$ of bounded continuous functions vanishing at infinity, in which $\mathscr{D}$ is dense. Hence (2.2) implies

$$
\mathrm{d} \mu_{0}=e^{\beta \phi} \mathrm{d} \mu_{\beta} .
$$

Let $\chi$ be the characteristic function of $(0, \infty)$ and set

$$
\mathrm{d} \mu=\chi \mathrm{d}_{\mu_{0}}+(1-\chi) \mathrm{d}_{\mu_{\beta}} .
$$

It is a finite complex measure. Let

$$
g_{\alpha}(p)=e^{-a p p} \chi(p)+e^{(\beta-\alpha) p}(1-\chi(p)),
$$

which is a bounded continuous function if $0 \leq \alpha \leq \beta$. Therefore

$$
\mathrm{d} \mu_{a}=g_{\alpha} \mathrm{d} \mu
$$

is a finite complex measure and

$$
\mathrm{F}(t+i \alpha) \equiv \int e^{i p t} \mathrm{~d} \mu_{\omega}(p)
$$

is a bounded continuous function of $t$ and $\alpha$ for $-\infty<t<+\infty$, $0 \leq \alpha \leq \beta$. From (2.10), (2.11), (2.12), we see that (2.4) is satisfied. For $0<\alpha<\beta, e^{i t p} g_{\alpha}(p)$ satisfies the Cauchy-Riemann relation with respect to $t+i \alpha$ in the topology of $\mathcal{C}_{0}$ and hence $\mathrm{F}(t+i \alpha)$ is holomorphic in $t+i \alpha$ for $0<\alpha<\beta$.

Remark 2. 3. The existence of $F$ satisfying (1), (2), (3) is equivalent to the KMS boundary condition. This is known except that the boundedness of $\mathrm{F}$ in the tube has not been treated in the literature.

Lemma 2. 4. Let $\quad \mathfrak{A}_{1} \equiv\left(\pi_{\varphi}(\mathfrak{R})\right)^{\prime \prime}, \quad \varphi_{1}(A)=\left(\Omega_{\varphi}, A \Omega_{\varphi}\right), \quad \tau_{1}(t) A$ $=U_{\varphi}(t) A U_{\varphi}(t)^{-1}\left(A \in \mathfrak{R}_{1}\right)$. Then $\varphi_{1}$ satisfies the KMS boundary condition with respect to $\mathfrak{A}_{1}$ and $\tau_{1}$, if $\phi$ satisfies the same with respect to $\mathfrak{A}$ and $\tau$.

Proof. We prove (2.2) for $\varphi_{1}, A \in \pi_{\varphi}(\mathfrak{Q})$ and $B \in \pi(\mathfrak{A})^{\prime \prime} . A$ similar argument will then yield (2.2) for general $A$ in $\pi_{\varphi}(\mathfrak{l})^{\prime \prime}$. Since $B=1$ obviously satisfies (2.2), we consider $B$ in the weak closure of $\pi_{\varphi}(\mathfrak{H})$. By the density theorem, it is enough to consider 
$B$ in the weak closure of the unit ball of $\pi_{\varphi}(\mathfrak{U})$.

Let $T$ be such that $\int_{|t|>T}\left|\tilde{f}_{0}(t)\right| \mathrm{d} t<\varepsilon$. Since $U_{\varphi}(t)$ is continous in $t$, we can find an open interval $I_{t}$ containing $t$ such that

$$
\left\|U_{\varphi}(t)^{-1} \pi_{\varphi}(A) * \Omega_{\varphi}-U_{\varphi}\left(t^{\prime}\right)^{-1} \pi_{\varphi}(A) * \Omega_{\varphi}\right\|<\varepsilon
$$

for any $t^{\prime} \in I_{t} . \quad A$ finite number of such $I_{t_{1}} \cdots I_{t_{n}}$ cover the compact interval $[-T, T]$. Let $N$ be the weak neighbourhood of $B$ defined by

$$
N=\left\{B^{\prime} ;\left|\left(U_{\varphi}\left(t_{j}\right)^{-1} \pi_{\varphi}(A) * \Omega_{\varphi},\left(B-B^{\prime}\right) \Omega_{\varphi}\right)\right|<\varepsilon, j=1 \cdots n\right\} .
$$

Then we have for $B^{\prime} \in N,\left\|B^{\prime}\right\| \leq 1$,

$$
\left|\int \varphi_{1}\left(A \tau(t)\left(B-B^{\prime}\right)\right) \tilde{f}_{0}(t) \mathrm{d} t\right| \leq 2|| A|| \varepsilon+3 \varepsilon \int\left|\tilde{f}_{0}(t)\right| \mathrm{d} t
$$

We have a similar equation for the right hand side of (2.2). Since (2.2) holds for $B^{\prime} \in \pi(\mathfrak{U})$, we have (2.2) for $B$ in the weak closure of the unit ball of $\pi(\mathfrak{U})$.

Q.E.D.

Corollary 2.5. The element of the center of $\pi_{\varphi}(\mathfrak{U})^{\prime \prime}$ is invariant under $\tau_{1}(t)$.

Proof. Since $U_{\varphi}(t) R U_{\varphi}(t)^{-1}=R$ holds for $R=\pi_{\varphi}(\mathfrak{Y})$, it holds for $R=\pi_{\varphi}(\mathfrak{U})^{\prime}$ and hence for $R=\pi_{\varphi}(\mathfrak{U})^{\prime \prime}$ and therefore for $R=$ the center of $\pi_{\varphi}(\mathfrak{U})^{\prime \prime}$. Thus

$$
\varphi_{1}\left(A \tau_{1}(t) B\right)=\varphi_{1}\left(\left[\tau_{1}(t) B\right] A\right)
$$

if $B$ is in the center of $\pi_{\varphi}(\mathfrak{A})^{\prime \prime}$. Lemma 2.2 implies the existence of a function $\mathrm{F}(\zeta)$ which is holomorphic for $0<\operatorname{Im} \zeta<\beta$ and continuous for $0 \leq \operatorname{Im} \zeta \leq \beta$. (2.4) and (2.18) implies, due to the edge of wedge theorem, that $\mathrm{F}(\zeta)$ is an entire function with period $i \beta$. Since $\mathrm{F}$ is bounded, it must be a constant. Since $\tau_{1}(t) B$ is in the center of $\pi_{\varphi}(\mathfrak{U})^{\prime \prime}$, we see that $\varphi_{1}\left(A_{1}\left[\tau_{1}(t) B\right] A_{2}\right)$ is constant of $t$ and hence $\tau_{1}(t) B=B$.

Remark 2. 6. This corollary can be proved also from (2.10) directly. Namely, $\mu_{0}=\mu_{\beta}$ and (2.10) imply $\mu_{0}=c \delta(p) \mathrm{d} p$, from which it follows that $\varphi(A \tau(t) B)$ is independent of $t$. 
This proof can be use to show that a state is invariant under $\tau(t)$, if it satisfies the KMS boundary condition (This is pointed out by $\mathrm{H}$. Miyata).

The equation (2.10) at $p=0$ implies that $\Omega_{\varphi}$ is a trace vector for $E(\{0\}) \pi_{\varphi}(\mathfrak{Q}) E(\{0\})$. This is used in [4].

\section{$\S 3$. Analyticity of Multiple Time Expectation Values}

Theorem 3.1. Let $\phi$ be a $\tau(t)$ invariant state of $\mathfrak{A}$ satisfying the KMS boundary condition. Let $A, B_{1}, \cdots, B_{n}$ be arbitrary $n+1$ elements of $\mathfrak{A}(n=1,2, \cdots)$. There exists a function $\mathrm{H}\left(\zeta_{1}, \cdots, \zeta_{n}\right)$ of $n$ complex variables such that

(1) $\mathrm{F}$ is holomorphic for

$$
0<\operatorname{Im} \zeta_{1}<\cdots<\operatorname{Im} \zeta_{n}<\beta
$$

(2) The boundary value of $\mathrm{F}$ for $\operatorname{Im} \zeta_{1}=\cdots=\operatorname{Im} \zeta_{j}=0, \operatorname{Im} \zeta_{j+1}$ $=\cdots=\operatorname{Im} \zeta_{n}=\beta$ in the distribution sense is the function

$$
\phi\left(\left[\tau\left(t_{j+1}\right) B_{j+1}\right] \cdots\left[\tau\left(t_{n}\right) B_{n}\right] A\left[\tau\left(t_{1}\right) B_{1}\right] \cdots\left[\tau\left(t_{j}\right) B_{j}\right]\right)
$$

where $j=0, \cdots, n$ and $t_{k}=\operatorname{Re} \zeta_{k}$.

Proof. Let us consider the Fourier transform of (3.2) in distribution sense :

$$
\begin{aligned}
& f_{j}\left(p_{1}, \cdots, p_{n}\right)=\int \phi\left(\left[\tau\left(t_{j+1}\right) B_{j+1}\right] \cdots\left[\tau\left(t_{n}\right) B_{n}\right] \times\right. \\
& \left.\quad \times A\left[\tau\left(t_{1}\right) B_{1}\right] \cdots\left[\tau\left(t_{j}\right) B_{j}\right]\right) e^{-i\left(p_{1} t_{1}+\cdots+p_{n} t_{n}\right) \times} \\
& \quad \times \mathrm{d} t_{1} \cdots \mathrm{d} t_{n} /(2 \pi)^{n}
\end{aligned}
$$

(2.2) implies

$$
f_{j+1}\left(p_{1}, \cdots, p_{n}\right)=e^{\beta p_{j+1}} f_{j}\left(p_{1}, \cdots, p_{n}\right) .
$$

Let $\chi_{j}$ be the characteristic function of the following region

$$
\begin{array}{r}
B_{j}=\left\{\left(p_{1}, \cdots, p_{n}\right) ; p_{k}+p_{k+1}+\cdots+p_{j}>0, \quad k=1, \cdots, j\right. \\
\left.p_{j+1}+\cdots+p_{k}<0, \quad k=j+1, \cdots, n\right\}
\end{array}
$$

where $j=0, \cdots, n$. Let $g$ be a nonnegative function in the class $\mathscr{D}$ such that 


$$
\int g\left(p_{1}, \cdots, p_{n}\right) d p_{1} \cdots d p_{n}=1
$$

and $\chi_{j}{ }_{j}$ be the regularization of $\chi_{j}$ by $g$ :

$$
\chi_{j}^{g}\left(p_{1}, \cdots, p_{n}\right)=\int \chi_{j}\left(p_{1}-r_{1}, \cdots, p_{n}-r_{n}\right) g\left(r_{1}, \cdots, r_{n}\right) \mathrm{d} r_{1} \cdots \mathrm{d} r_{n} .
$$

If we denote a vector with the first $k$ components equal to 1 and the last $(n-k)$ components equal to 0 by $q_{k}(k=0, \cdots, n)$, then $\bar{B}_{j}=\left\{p ; \max _{k}\left(p, q_{k}\right)=\left(p, q_{j}\right)\right\}$. From this we see that $\bigcup_{j=0}^{n} \bar{B}_{j}$ is the entire space and $\bar{B}_{j} \cap \bar{B}_{k}$ is in the plane orthogonal to $q_{j}-q_{k}$ if $j \neq k$, namely $\operatorname{dim} \bar{B}_{j} \cap \bar{B}_{k}<n$. Hence

$$
\sum_{j=0}^{n} \chi_{j}^{g}=1 .
$$

Let us define the following distribution

(3. 9) $\mathrm{H}\left(p_{1}, \cdots, p_{n} ; t_{1}+i \alpha_{1}, \cdots, t_{n}+i \alpha_{n}\right)$

$$
\begin{aligned}
= & \sum \chi_{j}^{g}\left(p_{1}, \cdots, p_{n}\right) h_{j}\left(p_{1}, \cdots, p_{n} ; \alpha_{1}, \cdots, \alpha_{n}\right) f_{j}\left(p_{1}, \cdots, p_{n}\right) \\
& \exp i \sum_{j=1}^{n} t_{j} p_{j}
\end{aligned}
$$

where

(3.10) $h_{j}\left(p_{1} \cdots p_{n} ; \alpha_{1} \cdots \alpha_{n}\right)=\exp \left\{\sum_{k=j+1}^{n}\left(\alpha_{k+1}-\alpha_{k}\right)\left(p_{j+1}+p_{j+2}+\cdots+p_{k}\right)\right.$

$$
\left.-\sum_{k=1}^{j}\left(\alpha_{k}-\alpha_{k-1}\right)\left(p_{k}+p_{k+1}+\cdots+p_{j}\right)\right\}
$$

and $\alpha_{0} \equiv 0, \alpha_{n+1} \equiv \beta$. If

$$
0<\alpha_{1}<\cdots<\alpha_{n}<\beta,
$$

then (3.10) implies that $h_{j}$ decreases exponentially whenever $\left(p, q_{j}-q_{l}\right)$ tends to $+\infty$ for one $l$. On the other hand the part of $B_{j}$, in which $\left(p, q_{j}-q_{l}\right)<R$ for all $l$ and a fixed $R>0$, is compact. Hence

$$
\exp i\left(p_{1} t_{1}+\cdots+p_{n} t_{n}\right) h_{j}\left(p_{1} \cdots p_{n} ; \alpha_{1} \cdots \alpha_{n}\right) \chi_{j}^{g}\left(p_{1} \cdots p_{n}\right)
$$

is in the class $\mathcal{S}$ and satisfies the Cauchy-Riemann relation with respect to each $t_{k}+i \alpha_{k}$. We now define 


$$
\mathrm{F}\left(\zeta_{1} \cdots \zeta_{n}\right)=\int \mathrm{H}\left(p_{1} \cdots p_{n} ; \zeta_{1} \cdots \zeta_{n}\right) \mathrm{d} p_{1} \cdots \mathrm{d} p_{n}
$$

which is holomorphic for $\zeta$ satisfying (3.1). Furthermore, the Fourier transform of the boundary value of $\mathrm{F}$ for $\operatorname{Im} \zeta_{1}=\cdots=\operatorname{Im} \zeta_{j}$ $=0, \operatorname{Im} \zeta_{j+1}=\cdots=\operatorname{Im} \zeta_{n}=\beta$ becomes

$$
\sum \chi_{k}^{g}\left(p_{1} \cdots p_{n}\right) h_{k}\left(p_{1} \cdots p_{n} ; \alpha_{1} \cdots \alpha_{n}\right) f_{k}\left(p_{1} \cdots p_{n}\right)
$$

where $\alpha_{1}=\cdots=\alpha_{j}=0, \alpha_{j+1}=\cdots=\alpha_{n}=\beta$. From (5.10) we have

$$
h_{k}\left(p_{1} \cdots p_{n} ; \alpha_{1} \cdots \alpha_{n}\right)=\left\{\begin{array}{ccc}
\exp \beta\left(p_{k+1}+\cdots+p_{j}\right) & \text { if } & k<j \\
1 & \text { if } & k=j \\
\exp -\beta\left(p_{j+1}+\cdots+p_{k}\right) & \text { if } & k>j
\end{array}\right.
$$

Hence, from (3.4), we have

$$
h_{k}\left(p_{1} \cdots p_{n} ; \alpha_{1}, \cdots \alpha_{n}\right) f_{k}\left(p_{1} \cdots p_{n}\right)=f_{j}\left(p_{1} \cdots p_{n}\right)
$$

for all $k$. By using (3.8), we see that the boundary value in question is (3.2).

Remark 3.2. (i) The above theorem and its proof are stated in a form which holds for Wightman fields. The next theorem uses the fact that $A$ and $B_{i}$ are bounded operators. (ii) In the discussion of the analyticity, it is more symmetric to consider

$$
\varphi\left(A_{1}\left(t_{1}\right) \cdots A_{n}\left(t_{n}\right)\right)
$$

on the space $\left\{\left(t_{1} \cdots t_{n}\right) \bmod (1, \cdots, 1)\right\}$. The step function $\chi_{j}$ can be written in terms of the edge vectors of the simplicial domain in question. For such a technique, see generalized $\theta$ function introduced in [5].

Theorem 3. 3. The function $F$ in Theorem 3.1 is continuous and bounded in the closure of the simplicial tube domain (3.1).

Proof. We investigate each summand more closely. By definition (3.3), we have

$$
\left\{\begin{array}{c}
\int f_{k}\left(p_{1} \cdots p_{n}\right) \exp i\left\{\sum\left(t_{l}+s_{l}\right) p_{l}\right\} d p_{1} \cdots d p_{n} \\
=\left(\Omega_{\varphi}, Q_{k+1} U_{\varphi}\left(s_{k+2}-s_{k+1}\right) Q_{k+2} \cdots Q_{n} U_{\varphi}\left(-s_{n}\right) Q_{0} U_{\varphi}\left(s_{1}\right)\right. \\
\left.Q_{1} U_{\varphi}\left(s_{2}-s_{1}\right) \cdots U_{\varphi}\left(s_{k}-s_{k-1}\right) Q_{k} \Omega_{\varphi}\right) \\
Q_{l}=\pi_{\varphi}\left[\tau\left(t_{l}\right) B_{l}\right], Q_{0}=\pi_{\varphi}[A] .
\end{array}\right.
$$


On the other hand

$$
\begin{aligned}
& \chi_{k}\left(p_{1} \cdots p_{n}\right)=\prod_{i=1}^{k} \theta\left(p_{i}+\cdots+p_{k}\right) I_{j=k+1}^{n} \theta\left(-\left(p_{k+1}+\cdots+p_{j}\right)\right) \\
& h_{k}\left(p_{1} \cdots p_{n} ; \alpha_{1} \cdots \alpha_{n}\right) \\
& \quad=\prod_{i=1}^{b} e^{-\left(\alpha_{i}-\alpha_{i-1}\right)\left(p_{i}+\cdots+p_{k}\right)} \prod_{j=k^{+1}}^{n} e^{\left(\alpha_{j+1}-a_{j}\right)\left(p_{k+1}+\cdots+p_{j}\right)}
\end{aligned}
$$

where $\theta$ is the characteristic function for positive reals. We note that

$$
\begin{aligned}
& \sum t_{l} p_{l}=t_{1}\left(p_{1}+\cdots+p_{k}\right)+\left(t_{2}-t_{1}\right)\left(p_{2}+\cdots+p_{k}\right)+\cdots \\
& \quad+\left(t_{k}-t_{k-1}\right) p_{k}-\left(t_{k+2}-t_{k+1}\right) p_{k+1}-\left(t_{k+3}-t_{k+2}\right)\left(p_{k+1}+p_{k+2}\right) \\
& \quad-\cdots-\left(t_{n}-t_{n-1}\right)\left(p_{k+1}+\cdots+p_{n-1}\right)+t_{n}\left(p_{k+1}+\cdots+p_{n}\right) .
\end{aligned}
$$

If we set

$$
\widetilde{\theta}(z)=\frac{1}{2 \pi} \int_{0}^{\infty} e^{-i z p} \mathrm{~d} p=\frac{1}{2 \pi i z}
$$

$$
\widetilde{g}\left(z_{1} \cdots z_{n}\right)=\int g\left(p_{1} \cdots p_{n}\right) \exp -i \sum_{l=1}^{n} z_{l} p_{l} \mathrm{~d} p_{1} \cdots \mathrm{d} p_{n}
$$

we have

$$
\begin{aligned}
& \text { (3. 22) } \int h_{k}\left(p_{1} \cdots p_{n} ; \alpha_{1} \cdots \alpha_{n}\right) \chi_{k}{ }^{g}\left(p_{1} \cdots p_{n}\right) \exp -i\left(\sum s_{l} p_{l}\right) \mathrm{d} p_{1} \cdots \mathrm{d} p_{n} /(2 \pi)^{n} \\
&= \widetilde{g}\left(z_{1} \cdots z_{n}\right) \tilde{\theta}\left(z_{1}\right) \tilde{\theta}\left(z_{2}-z_{1}\right) \cdots \tilde{\theta}\left(z_{k}-z_{k-1}\right) \tilde{\theta}\left(z_{k+2}-z_{k+1}\right) \cdots \\
& \cdots \tilde{\theta}\left(-z_{n}\right)
\end{aligned}
$$

where

$$
\begin{array}{cl}
z_{l}=s_{l}-i \alpha_{l}, & l=1 \cdots k \\
z_{l}=s_{l}-i \alpha_{l}+i \beta, & l=k+1 \cdots n .
\end{array}
$$

Combining (3.17) and (3.22), we obtain the following expression for the integral of the $k$ th term of (3.9).

$$
\begin{aligned}
& \int \tilde{g}\left(z_{1} \cdots z_{n}\right) \mathrm{d} s_{1} \cdots \mathrm{d} s_{n}\left(\Omega_{\varphi}, Q_{k+1} X\left(z_{k+2}-z_{k+1}\right) Q_{k+2} \cdots\right. \\
& \left.\quad \cdots Q_{n} X\left(-z_{n}\right) Q_{0} X\left(z_{1}\right) Q_{1} X\left(z_{2}-z_{1}\right) \cdots X\left(z_{k}-z_{k-1}\right) Q_{k} \Omega_{\varphi}\right)
\end{aligned}
$$

where

$$
X(z)=U_{\varphi}(\operatorname{Re} z) \tilde{\theta}(z) .
$$

For any testing function $\widetilde{g}$ in the class $\mathscr{D}$, we have 


$$
\int g(t-\zeta) X(t-\zeta) \mathrm{d} t=\int \widetilde{g}(q) \mathrm{d} q U^{+}(\zeta ; q)
$$

where $\operatorname{Im} \zeta \geqq 0, \operatorname{Re} \zeta=0$,

$$
\begin{aligned}
& g(t)=\int e^{-i t q} \tilde{g}(q) \mathrm{d} q, \\
& U^{+}(\zeta ; q)=\int_{q}^{\infty} e^{i \zeta^{\lambda}} \mathrm{d} E(\lambda),
\end{aligned}
$$

$E$ is the spectral projection of $U_{\varphi}(t)$.

The integral in (3.29) is ambiguous at the lower end but this ambiguity does not affect (3.27). If the lower end is $q \pm 0$, we denote $U_{ \pm}^{+}$. We define (3.29) as an average of $U_{+}^{+}$and $U_{-}^{+}$.

The expression (3.25) is then equal to

$$
\begin{aligned}
& \int g\left(p_{1} \cdots p_{n}\right)\left(\Omega_{\varphi}, Q_{k+1} U^{+}\left(\zeta_{k+1} ; q_{k+1}\right) Q_{k+2}\right. \\
& \left.\quad Q_{n} U^{+}\left(\zeta_{n} ; q_{n}\right) Q_{0} U^{+}\left(\zeta_{0} ; q_{1}\right) Q_{1} \cdots U^{+}\left(\zeta_{k-1} ; q_{k}\right) Q_{k} \Omega_{\varphi}\right) \mathrm{d} p_{1} \cdots \mathrm{d} p_{n}
\end{aligned}
$$

where

$$
\begin{gathered}
\zeta_{l}=i\left(\alpha_{l+1}-\alpha_{l}\right) \quad l=0, \cdots, n \\
\alpha_{n+1}=\beta, \quad \alpha_{0}=0 \\
q_{l}= \begin{cases}p_{l}+\cdots+p_{k} & \text { if } l \leqq k \\
-\left(p_{k+1}+\cdots+p_{l}\right) & \text { if } l>k .\end{cases}
\end{gathered}
$$

We now take the limit of sequence $g=g^{(\nu)}$ such that $\int g^{(\nu)} \mathrm{d} p_{1} \cdots \mathrm{d} p_{n}$ $=1, g^{(\nu)} \geqq 0 ; g^{(\nu)}=0$ for $\sum p_{l}{ }^{2} \geqq(1 / \nu)$. Let $B\left(\sigma_{1} \cdots \sigma_{n}\right)$ be the region in which $\sigma_{l} q_{l}>0$ for all $l$, where $\sigma_{l}= \pm 1$. Assume that

$$
\mu_{k}\left(\sigma_{1} \cdots \sigma_{n}\right)=\lim _{\nu} \int_{B\left(\sigma_{1} \cdots \sigma_{n}\right)} g^{(\nu)} \mathrm{d} p_{1} \cdots \mathrm{d} p_{n} .
$$

Then the limit of (3.30) is

$$
\begin{aligned}
& \sum_{\sigma_{1} \cdots \sigma_{n}} \mu_{k}\left(\sigma_{1} \cdots \sigma_{n}\right)\left(\Omega_{\varphi}, Q_{k+1} U_{\sigma_{k+1}^{+}}^{+}\left(\zeta_{k+1} ; 0\right) Q_{k+2} \cdots\right. \\
& \quad \cdots Q_{n} U_{\sigma_{n}}^{+}\left(\zeta_{n} ; 0\right) Q_{0} U_{\sigma_{1}}^{+}\left(\zeta_{1} ; 0\right) Q_{1} \cdots \\
& \left.\quad \cdots U_{\sigma_{k}}^{+}\left(\zeta_{k-1} ; 0\right) Q_{k} \Omega_{\varphi}\right)
\end{aligned}
$$

where $\mu \geqq 0$ and $\sum \mu\left(\sigma_{1} \cdots \sigma_{n}\right)=1$.

In obtaining (3.36) we have used the fact that $U^{+}(\zeta ; q)$ $-U_{\sigma}^{+}(\zeta ; 0)$ strongly tends to zero as $q \rightarrow 0$ with $\sigma q>0$, and that $\left\|U^{+}(\zeta ; q)\right\|$ is bounded uniformly in $q$ in the neighbourhood of 0 . 
Since

$$
U_{\sigma}^{+}(\zeta ; 0)=\int_{\sigma 0}^{+} e^{i \lambda \zeta} \mathrm{d} E(\lambda)
$$

is bounded and continuous for $\operatorname{Im} \zeta_{l} \geqq 0$ (and holomorphic for $\operatorname{Im} \zeta_{l}>0$ ), (3.36) is bounded and continuous in $\operatorname{Im} \zeta_{l} \geqq 0$.

Corollary 3. 4. $\mathrm{F}$ is given by

$$
\mathrm{F}\left(\zeta_{1} \cdots \zeta_{n}\right)=\sum \mathrm{F}_{k}\left(\zeta_{1} \cdots \zeta_{n}\right)
$$

(3. 39) $\mathrm{F}_{k}\left(\zeta_{1} \cdots \zeta_{n}\right)=\sum_{\sigma_{1} \cdots \sigma_{n}} \mu_{k}\left(\sigma_{1} \cdots \sigma_{n}\right)\left(\Omega_{\varphi}, \pi_{\varphi}\left(B_{k+1}\right) U_{\sigma_{k+1}}^{+}\left(\zeta_{k+2}-\zeta_{k+1}\right) \cdots\right.$

$$
\cdots U_{\sigma_{n-1}}^{+}\left(\zeta_{n}-\zeta_{n-1}\right) \pi_{\varphi}\left(B_{n}\right) U_{\sigma_{n}}^{+}\left(i \beta-\zeta_{n}\right) \pi_{\varphi}(A) U_{\sigma_{1}}^{+}\left(\eta_{1}\right)
$$

$$
\left.\pi_{\varphi}\left(B_{1}\right) U_{\sigma_{2}}^{+}\left(\zeta_{2}-\zeta_{1}\right) \cdots U_{\sigma_{k}}^{+}\left(\zeta_{k}-\zeta_{k-1}\right) \pi_{\varphi}\left(B_{k}\right) \Omega_{\varphi}\right) \text {, }
$$

where

$$
U_{\sigma}^{+}(\zeta)=\int_{\sigma 0}^{\infty} e^{i \zeta \lambda} \mathrm{d} E(\lambda)
$$

and $\mu_{k}\left(\sigma_{1} \cdots \sigma_{n}\right)$ is the volume of those part of the ball $p_{1}{ }^{2}+\cdots+p_{n}{ }^{2}=K$ of the unit volume which is defined by $\sigma_{l} q_{l}{ }^{(k)}>0, q_{l}{ }^{(k)}=p_{l}+\cdots+p_{k}$ for $l \leqq k, q_{l}{ }^{(k)}=-\left(p_{k-1}+\cdots+p_{l}\right)$ for $l>k$.

Proof. This follows from (3.36) where we take as $g^{(\nu)}$ a function obtained by smoothly cutting off tails of

$$
C(\nu) \exp -\nu \sum_{l=1}^{n}{p_{l}}^{2} .
$$

Remark 3.5. If we insert a formal expression

$$
U_{?}^{+}(\zeta)=\frac{1}{2 \pi i} \int U_{\varphi}(t) \frac{\mathrm{d} t}{t-\zeta} \quad(\operatorname{Im} \zeta>0)
$$

into (3.38) and (3.39), we obtain an unsubtracted form of the Bergman Weil formula.

Remark 3. 6. As a special case of $n=1$, we obtain

$$
F(\zeta)=\left(\Omega_{\varphi}, Q_{b} U^{+}(i \beta-\zeta) Q_{a} \Omega_{\varphi}\right)+\left(\Omega_{\varphi}, Q_{a} U^{+}(\zeta) Q_{b} \Omega_{\varphi}\right)
$$

where

$$
U^{+}(\zeta)=\int_{+_{0}}^{\infty} e^{i \zeta \lambda} \mathrm{d} E(\lambda)+\frac{1}{2} E(\{0\}) .
$$

By setting $\zeta=0$, we have 
(3. 44)

$$
\left(\Omega_{\varphi}, Q_{b} U^{+}(i \beta) Q_{a} \Omega_{\varphi}\right)=\left(\Omega_{\varphi}, Q_{a}\left(1-U^{+}(0)\right) Q_{b} \Omega_{\varphi}\right)
$$

\section{References}

[1] Haag, R., N. M. Hugenholtz and M. Winnik, Comm. Math. Phys. 5 (1966), 215-236.

[2] Hugenholtz, N. M., Comm. Math. Phys. 6 (1967), 189-193.

[3] Robinson, D., Comm. Math. Phys. 7 (1968), 337-348.

[4] Araki, H. and H. Miyata, Publ. RIMS Kyoto Univ. Ser. A, 4 (1968), 373-385.

[5] Araki, H., J. Math. Phys. 2 (1961), 163-177. 
